NOTA TÉCNICA

\title{
CAPACIDAD PRODUCTIVA DE CRUZAS DE VARIEDADES DE MAÍZ, DE POLINIZACIÓN LIBRE COMBINADAS CON HÍBRIDOS SIMPLES1
}

\author{
Alejandro Espinosa ${ }^{2}$, Margarita Tadeo ${ }^{3}$, Angel Piña ${ }^{3}$, Rafael Mart(nez ${ }^{3}$
}

\begin{abstract}
RESUMEN
Capacidad productiva de cruzas de variedades de polinización libre combinadas con híbridos simples de maíz. Dado la aceptación en los Valles Altos de México (2200-2600 msnm) de cinco variedades de polinización libre de maíz y la buena producción de cuatro híbridos, se consideró la alternativa de combinar las variedades de polinización libre con el H-34, así como otra cruza simple experimental. Las variedades Ixtlahuaca, Santiago Yeche y Acambay mejoraron de $37,3 \%$ a 20,8\% su producción al cruzarse con $\mathrm{H}-34$ no así Almoloya de Juárez y V-23. La otra cruza simple denominada 417, coadyuvó a una mayor producción de Ixtlahuaca en 29,1\%; cuando se combinó H-33 x 417, rindió 9728 $\mathrm{kg} / \mathrm{ha}$ superior en $7 \%$ a $9088 \mathrm{kglha}$ de $\mathrm{H}-33$; en cambio VS22 x 417 rindió muy similar a VS-22. Los resultados indican que podrían aprovecharse el H-34 y tal vez otras cruzas simples para combinarse con variedades de polinización libre, con lo cual se logra cierto nivel de heterosis y se facilita la producción de semillas, pero con base a la respuestas diferenciales, debería evaluarse previo a cualquier combinación.
\end{abstract}

\begin{abstract}
Potential productivity of crosses of varieties of open pollination with simple hybrids of corn. Given the acceplance, in the high valleys of Mexico -2200 to $2600 \mathrm{mts}$. over sea level-, of free-pollination strains and the high productivity of hybrids, we considered the alternative of combining these frec-pollination strains with the H-34, thus obtaining another experi-mental single hybrid and our goal was to determine its potential productivity level. The strains known aas Ixtlahuaca, Santiago Yeche and Acambay improved from $37,3 \%$ to $20,8 \%$ their productivity when crossed with H-34, such was not the case with the strains Almoloya de Juarez and V-23. The other single hybrid was named 417 , and it led to aHigher productivity in Ixtlahuaca with $29.1 \%$. When we conbined $\mathrm{H}-33$ x 417 it yielded 9728 kglha, a $7 \%$ higher than that of $9088 \mathrm{~kg} / \mathrm{ha}$ with $\mathrm{H}-33$. The results of VS-22x 417 were very similartothose with VS-22. The results indicated that we could use $\mathrm{H}-34$ and perhaps other single hybrids in combination with free-pollina-tion strains, thus obtaining a certain level of heterosis and facilitating seed production, but based on the different responses, this should beevaluated previous to any combination be performed.
\end{abstract}

\section{INTRODUCCION}

En los Valles Altos de México la adopción de semilla mejorada de maíz es escasa. Aún cuando los últimos cinco años se ha incrementado, se estima que apenas rebasa el 5\% de la superficie que se siembra con este cultivo. Por otra parte existen más de 300 mil hectáreas de maíz con riego y/o buenas condiciones de humedad. En otras 400 mil hectáreas sería conveniente el uso de variedades sintéticas y/o de polinización libre. Sin embargo se recomienda el uso de los híbridos H-28, $\mathrm{H}-30, \mathrm{H}-33, \mathrm{H}-34$ y las variedades V-23, VS-22, Ixtlahuaca, S. Yeche, Acambay, Almoloya de Juárez, etc. para ambas condiciones y para aprovechar las más favorables deberían emplearse los híbridos, pero no hay consenso en esta concepción estratégica por parte de los investigadores e instituciones que realizan investigaciones en esa región.

Durante varios años en el Instituto Nacional de Investigaciones Forestales y Agropecuarias (INIF AP), se recomendaron híbridos de escaso nivel de endogamia y de cruza doble lo cual en el fondo teórico se acercan a lo que sería una variedad de polinización libre. Desde los años cuarenta hasta los ochentas se mantuvo la estrategia de híbridos dobles, en los setentas se trató de obtener variedades mejoradas a partir de aplicar selección a materiales nativos, de esta manera se

\footnotetext{
1 Presentado en la XLII Reunión Anual del PCCMCA en El Salvador, 1996.

2 CEVAMEX, INIFAP, SARGD ,FES Cuautitlán, UNAM. México.

3 FES Cuautitlán, UNAM, México.
} 
obtuvieron: V -23, V-25, V-26A, V-29, VS-22, etc. Otras instituciones y universidades también han generado variedades mejoradas de polinización libre como ICAMEX que liberó: IxtIahuaca, Acambay, Almoloya de J., S. Yeche, etc.

La discrepancia en el tipo de variedades recomendadas se fundamenta en la poca adopción por parte de los agricultores de la semilla mejorada, generalmente la semilla de maíces nativos es de tamaño muy grande y precio menor a los híbridos, razón por la cual, los agricultores al conocer los maíces híbridos desconfían por el tamaño pequeño de la semilla, lo que fue más crítico cuando se liberó la primera cruza simple para los Valles Altos de México, el híbrido H-34, que participaba como cruza simple, en la conformación de varias cruzas dobles, ambas líneas del H-34 tenían aceptable productividad, (2500 kg/ha) (Espinosa, 1990), pero de manera lógica se ven afectadas fácilmente por cambios en el ambiente, reduciéndo la producción. El híbrido simple supera a las variedades de polinización libre en productividad, pero su fenotipo es muy similar a los maíces nativos, lo que hace que la ventaja observada en la báscula, tuviera poco impacto dentro de los agricultores vecinos. Finalmente el H-34 se dejó de producir por la limitada productividad de sus líneas al ser manejadas en programas de producción con agricultores cooperantes.

En lugar del H-34 se liberó en 1992 el híbrido doble denominado $\mathrm{H}-33$, en el cual participa como progenitor femenino el $\mathrm{H}-34$, las empresas semilleras prefieren este material, por su alta redituabilidad al incrementarse la semilla sobre una cruza simple rendidora (Espinosa, 1993a).

Hasta ahora ni los híbridos dobles y el H-34 (simple), ni las variedades mejoradas de polinización libre han tenido el éxito necesario que facilite el uso extensivo. Un argumento que se maneja es que no se acostumbra a la gente que cultiva maíz a la necesidad de comprar su semilla cada año, o que el precio de ésta es muy elevado, al grado de ser México un país donde la semilla se vende a muy alto precio (López-Pereira y Filippello, 1994 ).

Se reconoce que los híbridos con el polen que diseminan, han tenido influencia favorable en elevar la productividad, por otra parte la Universidad Nacional Autónoma de México (UNAM) dispone de nuevos híbridos de maíz de conformación trilineal que estratégicamente podrían ser un paso intermedio de adopción hacia híbrido s simples (Espinosa et al, 1995). Otra alternativa para promover un mayor uso tal vez sería la combinación de materiales de P.L. con cruzas simples que mejorarían su potencial productivo.

Además de cierto nivel de heterosis que podrían ganar las combinaciones, el esquema permitiría la producción de semilla a un precio inferior a los híbridos convencionales y tal vez el manejo por parte de agricultores para producir su propia semilla.

En este trabajo se presentan resultados obtenidos con la evaluación de híbridos no convencionales, es decir que se generaron al combinar cruzas simples de maíz (H-34 y 417) con siete variedades mejoradas de polinización libre y un híbrido doble, el objetivo del estudio fue definir el potencial productivo de este tipo de materiales y analizar la perspectiva de su uso en la producción de semillas.

\section{MATERIALES Y MÉTODOS}

En el ciclo primavera-verano de 1993 fueron evaluados en el Campo Experimental de la Facultad de Estudios Superiores Cuautitlán, UNAM, localizado a 2240 msnm, los materiales de maíz que se muestran en el Cuadro 1.

El diseño utilizado fue de bloques completos al azar con cuatro repeticiones, la parcela total estuvo

Cuadro 1: Materiales de maíz, evaluados en el campo Experimental de la Facultad de Estudios Superiores, Cuautitlán UNAM. México, 1993.

\begin{tabular}{lcc}
\hline Variedades P.L. & Híbridos simples & Híbridos no convencionales \\
\hline Ixtlahuaca & H-34 & Ixtlahuaca $\times$ H-34 \\
Almoloya & 417 & Ixtlahuaca $\times 417$ \\
S. Yeche & & Almoloya $\times$ H-34 \\
Acambay & Híbrido doble & S. Yeche $\times$ H-34 \\
VS-22 & H-33 & Acambay $\times$ H-34 \\
V-23 & & VS-22 $\times 417$ \\
V-23 x H-34 & & H-33 $\times 417$ \\
\hline
\end{tabular}


constituida por cuatro surcos de cinco metros y la parcela útil de los dos surcos centrales.

Se utilizó una densidad de población de 60.000 plantas por hectárea. Las variables en estudio fueron: rendimiento al $14 \%$ de humedad, altura de planta y mazorca y floración masculina y femenina.

\section{RESULTADOS Y DISCUSIÓN}

Los análisis de varianza detectaron diferencias altamente significativas para rendimiento y sólo al nivel de $0,05 \%$ para altura de mazorca (Cuadro 2). La media de rendimiento del experimento fue de 7,980 $\mathrm{kg} / \mathrm{ha}$ y el Coeficiente de Variación de 7,7 \%.

El máximo rendimiento correspondió al híbrido simple H-34, con $9948 \mathrm{~kg} / \mathrm{ha}$, reflejo de su buena capacidad productiva. Este maíz fue liberado comercialmente para los Valles Altos de México en 1990, teniéndose que dejar de producir semilla en 1993 por el elevado precio de su semilla, basado en la limitada producción de sus progenitores (Espinosa, et al., 1995). El H-33 híbrido de cruza doble, que sustituyó comercialmente al H-34, se ubicó muy cerca de este último en rendimiento $(9088 \mathrm{~kg} / \mathrm{ha})$, lo que ratifica sus ventajas, como material comercial, ya que su semilla representa alta redituabilidad para las empresas semilleras que lo multiplican por ser híbrido doble (López-Pereira y Filippello, 1994 ). En el híbrido H-33 participa como cruza simple hembra el H-34, este material esta conformado por líneas de baja endogamia, por lo cual la disminución en rendimiento al pasar de cruza simple a doble no es fuerte (Espinosa, 1993b).

La variedad sintética VS-22 fue el material de polinización libre de mayor rendimiento $(9443 \mathrm{~kg} / \mathrm{ha})$, el resto de las variedades expresaron rendimientos por debajo de las nueve toneladas. Como se esperaba, los híbridos superaron a las variedades pero de acuerdo con el objetivo de este trabajo, se aprecia que las variedades Ixtlahuaca, Santiago Yeche y Acambay mejoraron en $37,3 \% ; 21,9 \%$ y $20,8 \%$ su producción al combinarse con el híbrido simple H-34 (Cuadro 1).

En el caso de Ixtlahuaca x H-34, este genotipo produjo $7030 \mathrm{~kg} / \mathrm{ha}$, habiendo una ganancia de 1912 $\mathrm{kg} /$ ha por efecto de la cruza; S. Yeche x H-34 rindió $8156 \mathrm{~kg} / \mathrm{ha}$ y la cruza influenció en una mejoría de $1468 \mathrm{~kg} / \mathrm{ha}$, en cambio Acambay x H-34 mejoró en $1503 \mathrm{~kg} / \mathrm{ha}$, con respecto a la variedad. Estos valores son importantes si se considera que al sembrarse este tipo de combinaciones en lugar de las variedades, se obtendría una fracción adicional de grano.

Cuadro 2. Resultados obtenidos con variedades de polinización libre de maíz en combinación con híbridos simples de Valles Altos de México. FESC-UNAM, 1993.

\begin{tabular}{|c|c|c|c|c|c|}
\hline \multirow[t]{2}{*}{ Genotipos } & \multirow{2}{*}{$\begin{array}{l}\text { Rend. } \\
\text { (kg/ha) }\end{array}$} & \multirow{2}{*}{$\begin{array}{c}\% \text { V.S. } \\
\text { variedad P.L. }\end{array}$} & \multicolumn{2}{|c|}{ altura de } & \multirow{2}{*}{$\begin{array}{l}\text { floración } \\
\text { Masc. (días) }\end{array}$} \\
\hline & & & planta & mazorca & \\
\hline Ixtlahuaca x H-34 & 7,030 & 137,3 & 285 & 198 & 83 \\
\hline Ixtlahuaca x 417 & 6,612 & 129,1 & 281 & 172 & 79 \\
\hline Ixtlahuaca & 5,118 & 100,0 & 296 & 188 & 80 \\
\hline Almoloya x H-34 & 7,175 & 98,5 & 301 & 200 & 83 \\
\hline Almoloya & 7,282 & 100,0 & 282 & 187 & 82 \\
\hline S. Yeche $x$ H-34 & 8,156 & 121,9 & 310 & 213 & 83 \\
\hline S. Yeche & 6,668 & 100,0 & 283 & 193 & 83 \\
\hline Acambay x H-34 & 8,716 & 120,8 & 296 & 210 & 83 \\
\hline Acambay & 7,213 & 100,0 & 297 & 206 & 81 \\
\hline $\mathrm{V}-23 \times \mathrm{H}-34$ & 7,472 & 99,3 & 296 & 199 & 85 \\
\hline $\mathrm{V}-23$ & 7,522 & 100,0 & 304 & 204 & 87 \\
\hline VS-22 x 417 & 9,425 & 99,8 & 286 & 198 & 82 \\
\hline VS-22 & 9,443 & 100,0 & 316 & 208 & 86 \\
\hline H-33 $\times 417$ & 9,728 & 107,0 & 287 & 181 & 82 \\
\hline $\mathrm{H}-33$ & 9,088 & 100,0 & 290 & 204 & 87 \\
\hline $\mathrm{H}-34$ & 9,948 & & 326 & 219 & 88 \\
\hline D.S.H. $(0,05)$ & 1,922 & & 47 & 40 & \\
\hline
\end{tabular}


Las variedades Almoloya de Juárez y V-23 no mejoraron la expresión de su rendimiento al combinarse con $\mathrm{H}-34$, pero su rendimiento fue muy similar al de las variedades sin combinarse. Lo anterior indica que si bien hubo combinaciones ventajosas, la cruzas de Almoloya y V-23 señalan que antes de hacer extensiva una nueva combinación, es necesario evaluarlas (Cuadro 1).

Con respecto a la cruza simple 417 , ésta coadyuvó a una mayor producción de Ixtlahuaca $(6612 \mathrm{~kg} / \mathrm{ha})$ en $29,1 \%$ con respecto a la misma variedad sin cruzar; Al combinarse el híbrido doble $\mathrm{H}-33$ por la cruza simple 417 , rindió $9.728 \mathrm{~kg} / \mathrm{ha}$ que es superior en $7 \%$ a la producción de H-33 (9.088 kg/ha).

La variedad sintética VS-22 no exhibió ventajas en producción al cruzarse con el híbrido 417, siendo 99,8\% la expresión del rendimiento de la cruza con respecto a la variedad.

Los resultados indican que en varios casos se generaron excedentes en producción por efecto de las cruzas y en aquellos casos donde no hubo ganancia tampoco hubo reducción en la capacidad productiva. Por lo cual se podría deducir que es factible aprovechar este tipo de combinaciones, lo que ejemplifica tal vez la bondad adicional que han aportado las variedades mejoradas al difundirse su polen en parcelas de agricultores vecinos. Lo cual implica un beneficio difícil de cuantificar del mejoramiento genético a la agricultura mexicana aún cuando los materiales mejorados se utilicen en baja escala.

En relación a las alturas de planta y mazorca, se observó que al combinarse con H-34, la mayoría de las variedades incrementaron su porte de planta y colocación de la mazorca ya que H-34 es de porte alto. En cambio con la cruza simple 417 disminuyó la altura de planta y mazorca porque esta cruza es de porte bajo, lo cual indica que podría mejorarse alguna característica, teniendo cIaro lo que se desea incorporar con el híbrido combinador.

\section{LITERATURA CITADA}

ESPINOSA C., A. 1990. Disponibilidad de tecnología de producción de semillas, investigación en marcha y a futuro para maíz en México. In: Memorias de II Reunión Nacional de Semillas, Sociedad Mexicana de Fitogenética, SOMEFI. pp. 127-144, Chapingo, México.

ESPINOSA C., A. 1993a. Tecnología de producción de semillas del híbrido de maíz H-33 para Valles Altos. In: Agronegocios en México. 2(12):33-39.

ESPINOSA C., A. 1993b. Tecnología de producción de semillas de maíz en México. In: Memorias del Primer Simposium Internacional, Cuarto Nacional" El maíz en la década de los 90". Zapopan, Jalisco, México.

ESPINOSA c., A.; TADEO M; PIÑA A; MARTINEZ, R 1995. Generación de híbridos de maíz de Valles Altos de México y su empleo en Microempresas de Semillas. Agronomía Mesoamericana 6: 148-150.

LOPEZ-PEREIRA , M.A; FILIPPELLO, M. 1994. Maize seed industries, revisited: emerging roles of the public and private sectors. Part I of CIMMYT 1993/94 World maize facts and trends. Maize Seed Industries, Revisited: Emerging Roles of the Public and Private Sectors. México, D.F. 